\title{
Single Cell Analysis and Circulating Tumor Cells in Prostate Cancer: Experience of an Observership at Cold Spring Harbor Laboratory, CSH and New York Presbyterian Hospital (Cornell Campus), New York
}

\author{
Asian Pac J Cancer Prev, 16 (14), 6173-6174
}

\section{Dear Editor}

Prostate cancer has emerged as a major public health problem worldwide (Pandey and Chandravati, 2013). Prostate tumors shed circulating tumor cells (CTCs) into the bloodstream of patients diagnosed with metastatic, castration-resistant prostate cancer (CRPC) and Hormone Refractory Prostate Cancer (HRPC); CTC counts predict overall survival and provide an efficient framework for multi-scale analysis of fluid biopsies for quantification as well as monitoring of prostate cancer in individual patients of diverse ethnicities (Ozkumur et al., 2013; Wang et al., 2011; Turker et al., 2013).

This letter to editor aims to briefly comment on the predictive and prognostic therapeutic potential of CTCs in tailor-made personalized treatment strategies in prostate cancer management; the idea to draft the letter originated from the lead author's clinical research observership/ visit at Cold Spring Harbor Laboratory, CSH, and New York Presbyterian Hospital (Weill-Cornell campus), NY, USA. High content analyses of single CTCs have the potential to provide a non-invasive means of assessing prostate cancer progression in real time during the course of treatment, and to aid in patient-specific tailormade individualized therapy by monitoring phenotypic, physiological, morphometric, genotypic and protein expression alterations in real time occurring in response to targeted therapy (Dago et al., 2014).

Moreover, highly rearranged copy number variation (CNV) profiles and androgen receptor (AR)+/- phenotypes in distinct CTC subpopulations may further predict the therapeutic response using pharmacodynamics and/or pharmacogenomics strategies in animal models (murine models of prostate cancer) and prostate cancer human cohorts of American (Whites, Hispanic, Caucasian, African American) and Asian (Indian, Korean, Chinese, Japanese) ethnicities at the population level(s). Recently, genomic changes based on array $\mathrm{CGH}$ and limited sequencing have been reported on CTCs isolated with the CellSearch system and the High DefinitionCTC (HD-CTC) enumeration method as well as the NanoVelcro Chip assay (Lu et al., 2013). Interestingly, CTCs expressing genes related to epithelial-mesenchymal transition (EMT), viz. Wnt 5a, FOXP3, IGF1/2, EGFR and
TGF- $\beta 3$, have been suggested to be strong predictors of metastatic prostate cancer (Chen et al., 2013).

Accurate isolation/characterization of CTCs using high content single cell analysis holds tremendous prognostic and predictive therapeutic potential in prostate cancer management in both the American and Asian Indian patient cohorts. Indeed, single cell analyses/CTC enumeration provides novel therapeutic clinically-relevant intervention points/patient-centric opportunities for personalized treatments with targeted inhibitors in specific subsets of prostate cancer patients stratified according to Gleason score/prostate specific antigen level and tumor stage/metastasis.

Future clinical research targeting tumor-specific CTC subpopulations may be tremendously beneficial in the successful design/validation of cost-effective, sensitive and specific patient-centric predictive and prognostic biomarkers in human cancers, including prostate cancer, thereby reducing the increasing burden of cancers among ethnically disparate populations.

\section{Acknowledgements}

Dr. Saumya Pandey (M.Sc., Ph.D.) acknowledges the academic/clinical research support of the reputed faculty/ staff at the Watson School of Biological Sciences, Cold Spring Harbor Laboratory, CSH, and Dr. Ashutosh Tiwari, Chair (former), James Buchanan Brady Foundation, Department of Urology at Weill Medical College of Cornell University, New York, NY, USA during prostate cancer-related collaborative research discussions(s), and Dr. Gita Khanna of Ajanta Hospital and IVF Centre, Lucknow, U.P., India for providing the academic/clinical infrastructure for performing comprehensive Pubmed literature search during preparation of this manuscript.

\section{References}

Chen CL, Mahalingam D, Osmulski P, et al (2013). Single-cell analysis of circulating tumor cells identifies cumulative expression patterns of EMT-related genes in metastatic prostate cancer. Prostate, 73, 813-26.

Dago AE, Stepansky A, Carlsson A, et al (2014). Rapid phenotypic and genomic change in response to therapeutic 
pressure in prostate cancer inferred by high content analysis of single circulating tumor cells. PLoS One, 9, 101777.

Lu YT, Zhao L, Shen Q, et al (2013). NanoVelcro Chip for CTC enumeration in prostate cancer patients. Methods, 64, 144-52.

Ozkumur E, Shah AM, Ciciliano JC, et al (2013). Inertial focusing for tumor antigen-dependent and -independent sorting of rare circulating tumor cells. Sci Transl Med, 5, 17947.

Pandey S and Chandravati (2013). Robotic prostatectomy in urological surgery: an observership at Weill Medical College of Cornell University, New York. Asian Pac J Cancer Prev, 14, 4945 .

Turker I, Uyeturk U, Sonmez OU, et al (2013). Detection of circulating tumor cells in breast cancer patients: prognostic predictive role. Asian Pac J Cancer Prev, 14, 1601-7.

Wang FB, Yang XQ, Yang S, et al (2011). A higher number of circulating tumor cells (CTC) in peripheral blood indicates poor prognosis in prostate cancer patients-a meta-analysis. Asian Pac J Cancer Prev, 12, 2629-35.

\section{Saumya Pandey ${ }^{1,2 *}$, Anil Khanna ${ }^{1}$}

${ }^{1}$ Ajanta Hospital and IVF Centre, Lucknow, U.P., India, ${ }^{2}$ University of Texas Medical Branch, Galveston, TX, USA *For correspondence:drsaumyapandey6@gmail.com 